\title{
Pi Wei Xiang Biao Li and the Trajectory of Zuyangming
}

\author{
Electra Peluffo \\ Faculty of Medicine, Universidad de Valencia, Valencia, Spain \\ Email: acupuntura@electrapeluffo.com
}

Received 9 January 2014; revised 10 February 2014; accepted 23 February 2014

Copyright (C) 2014 by author and Scientific Research Publishing Inc.

This work is licensed under the Creative Commons Attribution International License (CC BY). http://creativecommons.org/licenses/by/4.0/

(c) (i) Open Access

\begin{abstract}
In this paper, it is studied the reason why Zuyangming (stomach meridian) travels the body through yin territory in contrast with the other eleven main meridians governed by the quantitative-qualitative yinyang synchronicity of meridians and territories to discover. Zuyangming meets this principle only partially (in face and leg) and that's why the reasons for this exception in the thoraco-abdominal trajectory are attempted to be deciphered here. Ancient traditional medical texts describe the internal and external routes of meridians and the relations between the spleen stomach pair, which $\mathrm{Su}$ Wen 8 transforms into spleenstomach, mentioning the membranous binding (connective tissue today) which unifies both viscera. In addition, the embryological studies of the human fetus provided information on yuan-source of life-and the development of tissues (fascia and membranes) during fetal organic evolution, allowing us to understand many of the classical ideas about life, health and disease. Reflections in this paper conclude that zuyangming internalizes its resplendent yang energy, and thus compensates for the predominant presence of yin energy (yin organs in yin territory), keeping the inter organic harmony of energy blows.
\end{abstract}

\section{Keywords}

Zuyangming; Zutaiyin; Stomach and Spleen Meridians; Membranes; Connective Tissue; Su Wen; Ling Shu; Shang Han Lun; Nan Jing

\section{Introduction}

The author started studying Chinese Medicine under Professor Ye Chenggu at Guan An Men Hospital in Beijing between 1965 and 1967, time during which the subject concerning this study already caught her attention. Subsequently, in 1982 she attended classes individually, at that same Hospital, under the direction of Professor Gao Lishan with whom she consulted the difference shown by the stomach meridian along its path. Dr Gao pointed 
out that the question itself revealed a clear understanding of the energetic physiology of the Chinese Medicine. However, it was only after long years of practice and studies on Classic Medicine text that the author was able to develop a hypothesis about why the stomach meridian does not follow the yinyang rule of territorial distribution.

\section{Working Hipothesis}

Ancient Chinese Medical texts provide detailed information, both theoretical and practical, on meridians and their trajectories; these data when elaborated, allow us to develop a hypothesis that explains the reasons why the stomach meridian path differs from the yinyang rule governing the energetic channels trajectories.

The Medicine Classics revealed the interorganic physical relationship through membranes (currently known as connective tissue) between the members of the pair linking spleen and stomach as shown in the internal path of the respective meridians and their functions, concepts accepted until now.

This work emphasizes the Chinese classic conception of energy and cosmic resonance, as well as the relationship between the yangming (the innermost of the yang energy levels) and taiyin (the outermost of yin levels) which shows the bond not only material, anatomical but also functional; being the principle of complementary opposition, so dear to Chinese thought, fully respected.

The thoraco abdominal cavity (anterior face of the body and therefore yin) is home to almost all the zang fu (organs and viscera) that give the area a greater yin energy charge, which according to our hypothesis, is sought to get counteracted by the presence of hollow and peristaltic yang bowels whose main representative is the stomach yangming, resplendent energy, energy that is internalized when getting in contact with taiyin.

\section{Material and Method}

Analytical reading of chapters deals with the subject in both ancient and modern translations-interpretations of Classics: Su Wen, Ling Shu, Nan Jing, Huai Nan Zi and Shang Han Lun, listed in References.

\section{Zuyangming Trayectory}

Stomach meridian, zuyangming, starts in the nose through which it goes up and down, covers the upper gums and teeth, comes in front of the ears and rises up to the scalp; this journey through the face allows it to include all the organs of the senses. It goes down to the neck (St.9) and shoulder along the horizontal branch of the jawbone and turns towards the spine to meet the yang meridians in GV.14. After that it reaches the clavicle, and from there one branch penetrates the anterior trunk and moves forward crossing the diaphragm so as to, in CV13, enter the stomach and keep descending until the groin among yin meridians (all of them with an ascending direction) and returning to yang areas, travelling along thigh, leg and foot $\left(2^{\text {nd }}\right.$ and $3^{\text {rd }}$ toe) (Figures $\left.1-3\right)$.

The twelve main meridians get together or pair up to form the six levels of energy that exchange their breaths. Out of this confluence six energetical dynamics are born, among them yangming-taiyin [1] spleenstomach which is the one before us today.

Zuyangming — stomach —is shining yang, radiant and being the innermost of all yang meridians in the six energetic levels concept, gets to contact coupled zutaiyin (spleen) to interchange their opposite and complementary energies.

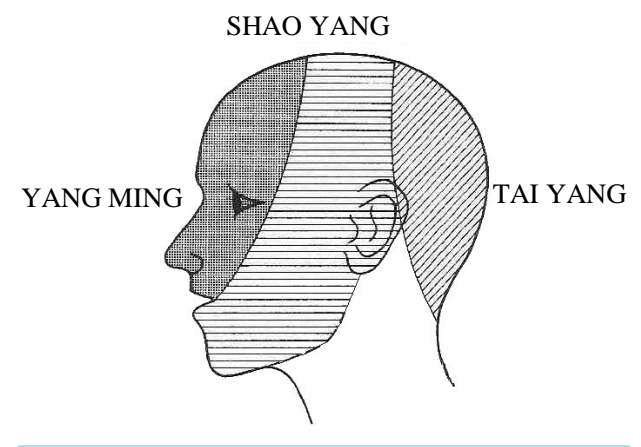

Figure 1. Head. Yang areas. 


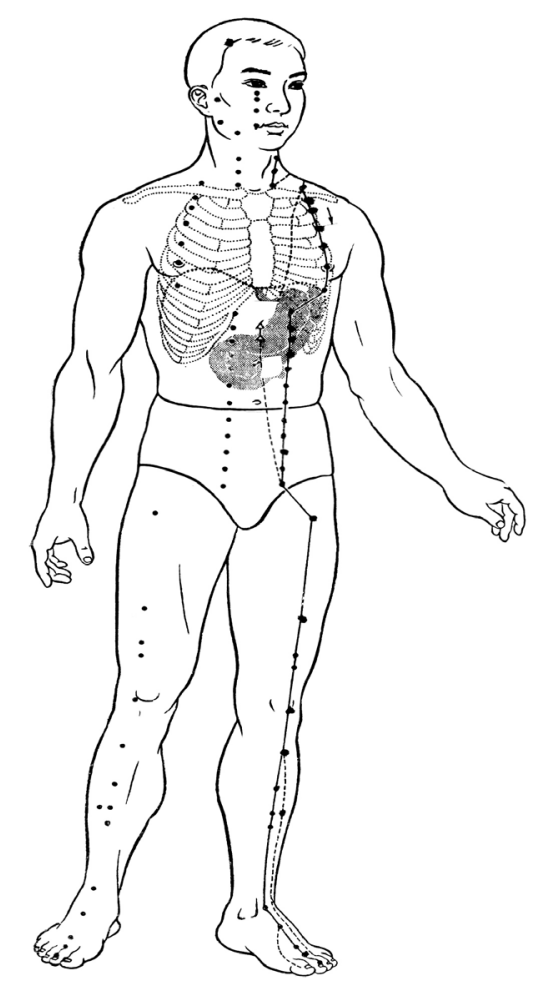

Figure 2. Zuyangming.

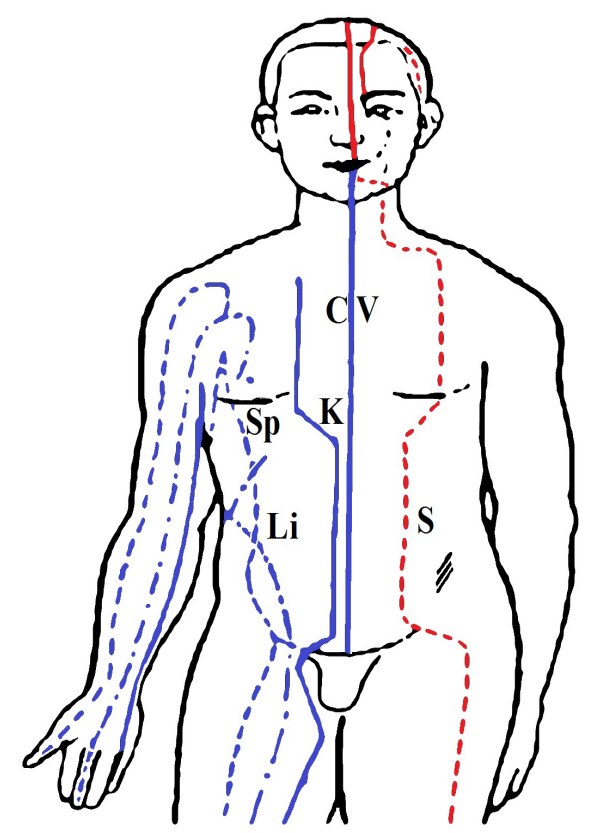

Figure 3. Left, zuyangming. Right, yin meridians.

\section{Internal Routes of Zuyangming and Zutaiyin}

The inner route of Zuyangming is told to us by Ling Shu 10: “... in Quepen (St.12), one branch descends along the thorax and spirally envelops the spleen. Another branch begins at the stomach exit and descends through the stomach lining until St.30 Qichong (also Qijie) [2]. When mentioning the lining of the stomach, is it referring to 
mesentery, hypodermic fascia?

Almost all texts accept Ling Shu version except Lei Jing Tu Yi (Classics of Categories) which details that the branch going down through the abdomen cover, descends slightly laterally to the kidney meridian, and begins at the same level as Huangshu Kid.16, right by the navel [3]. Both references correspond to yin transits.

There is a difference in language regarding the energetical relationship between an organ and its meridian and with coupled organ and meridian. If it is said that the meridian belongs or penetrates (shu) certain organ that means the meridian is completely rooted in it, in a reciprocal relation of mutual belonging. For instance, the liver and the liver meridian constitute a whole like a basin between a river and its lakes, inseparable.

The relationship between the inner trajectory of a meridian and the paired organ which it spirally envelopes (luo) is different. The connection is not so intimate any more, the river contours, envelopes a rock, it does not penetrate it: stomach spirally [4] envelopes around the spleen. And also spleen spirally envelopes (luo) stomach (Figures 4 and 5).

Wang Bing (710-804) in his Su Wen compilation says "zuyangming [5], stomach vessel, descends through the diaphragm, touches the stomach and envelopes the spleen...” This spiral connection as it is told in the Classics is established by membranes, sheaths. Su Wen 29 in its final lines describes the connection between spleen and stomach through tissues and membranes that keep the two organs attached so that body fluids jin ye, can circulate through both of them.

And thus, the density of the spleenstomach relationship is highlighted thanks to the membranes that gather them together with the same density of relationships on earth, so intimate they reach physical contact, and that enables the control of that which flows between those two membranes.

When the Emperor asks Su Wen 29 [6]-whether the jin ye actually go through this kind of tissue or membrane to reach the entire body and specially until the viscera, Qi Bo answers that zutaiyin (spleen) runs through the stomach and establishes a shu dependency relationship with the spleen (penetrates it) and a luo relationship with the throat (envelopes it). Thus, taiyin makes the qi circulate in the three yin levels: tai, shao and jueyin.

Zuyangming is the obverse movement biao, that is towards the outside, is the sea of the 5 zang and the $6 \mathbf{f u}$, responsible for the circulation of qi through the three yang levels: tai, shao and yangming.

Clear is the responsibility of spleenstomach in front of the six great meridians, three yin, and three yang and also in front of the twelve main meridians.

Spleen and stomach interact so as to share the circulatory function of the yin yang meridians because although it is assured in Su Wen 29 that both zang and fu get qi through the yangming (stomach makes jinyebody fluids-circulate) this would not happen without the intervention of the spleen, that makes this simultaneous yangyin movement irrigate the whole body.

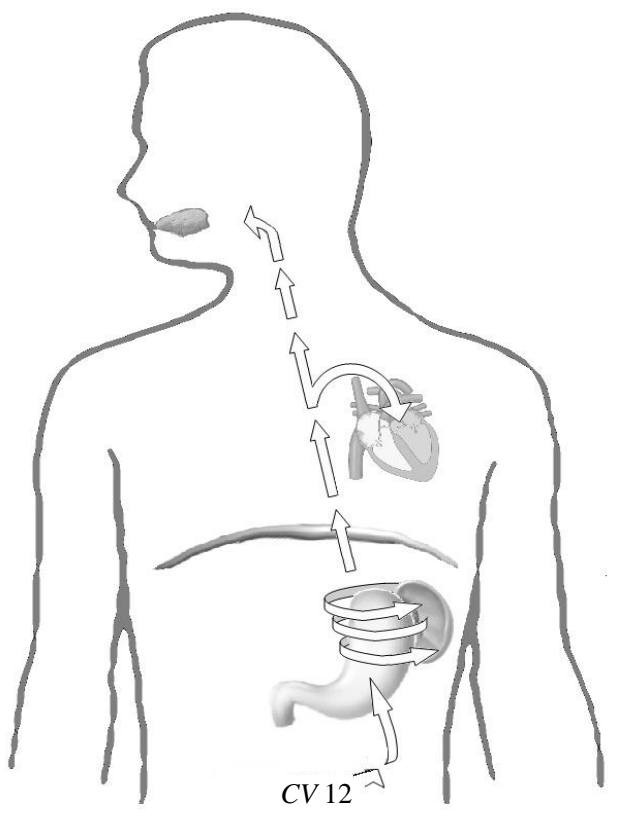

Figure 4. Internal route zutaiyin. 


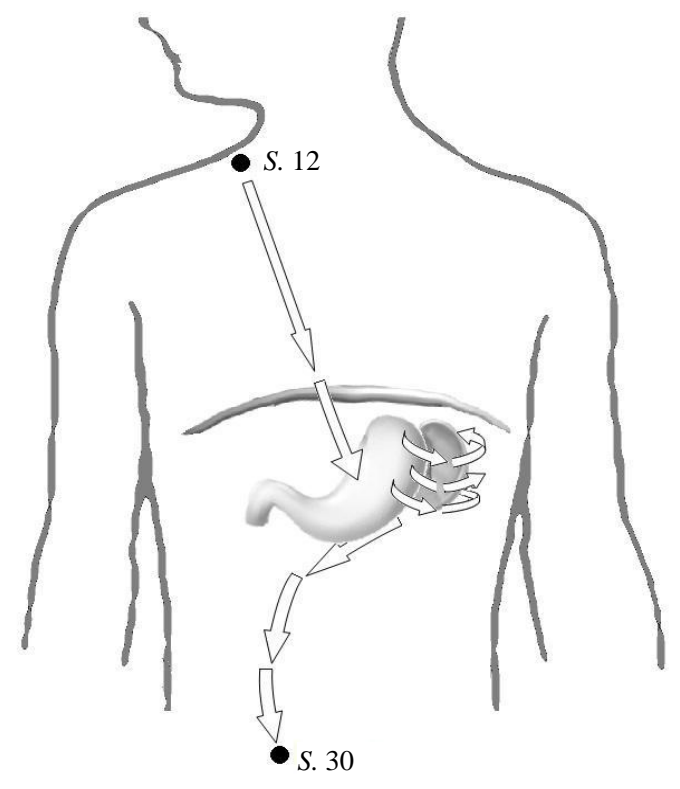

Figure 5. Internal route zuyangming.

\section{Membranes. Yuan: Source, Origin}

Chinese people paid attention to human embryology because they were interested in concepts such as "beginning, root, origin" ...many Chinese ideographs imply the notion of origin or root or basic part: yuan source, basic idea in the source theory in Ling Shu 1 and in Nan Jing 66. They wanted to know where things came from, the source or origin of life, and embryology gave them an essential information, because fetal organic development and its connective tissues, fascias, membranes allowed them to understand many of the classic ideas upon life, health and illness [7]. This evolution of the embryo was already announced in Huai Nan $\mathrm{Zi} 7$ [8] which describes the human being development based on a previous text, Guan Zi [9] from IV century BC where membranes and mesenteric fascias were not discussed as shown in Huai Nan Zi.

These mentions of the Classics show that, from ancient times, the anatomical presence of the interorganic membranes linking spleen and stomach together with their internal trajectories were well known.

The connective tissue is a versatile organic system, quite widespread and omnipresent which, being both well known and well analyzed by modern medicine, interconnects all parts of human body in each level, from the macro to microscopic one and can be found even in the simplest of the organelles, in each cell and in each texture or body framework.

Of greater importance for Eastern medicine-both theoretical and practical—are the now known properties these tissues show: to generate energy and to conduct it.

The Chinese conceived and sensed the energy of the qi watching the winds and their movements both in time and space.

Ganying, the cosmic resonance, the stimulus and the response to stimulus, is an essential ingredient in the Chinese thought. Resonances were not, are not abstract, there is always a material subtract so that they can occur. Joseph Needham [10] highlights the Chinese predilection for "action from a distance."

It is registered that in China in the year 132 an apparatus to detect earthquakes was designed, that device used the concept of action from a distance, the resonance already mentioned in Huai Nan $\mathrm{Zi}$ chapter 6 as a purely vibrational phenomenon of the qi [11]. Knowing these data, it is not noteworthy that Chinese attributed field effects — to generate and to drive energy — to the connective tissue and its numerous variants in the body.

\section{Western Conception, Eastern Conception}

Let us start from the acceptance that Chinese medicine is a complete and independent medical system.

We the Western medicine doctors, are instructed (taught and learned) through topics of learning in which Anatomy, organs, materiality have an undoubted predominance. And by this, please do not understand that I am 
suggesting that Physiology should not be studied; on the contrary a lot of it, all of it should be studied! But we have to accept that the Western comprehension of the Eastern way of presenting the body and its functioning is always interfered by our outstanding organic references which, for instance, lead to translate the names of the meridians using organ denominations whereas the original Chinese medicine defines them using their topography (hand or foot), quality (yin yang) and quantity (tai, shao, jue). Confusion prevails, even admitting that Eastern concepts are difficult to translate and to explain especially if homologation with our way of thinking is sought [12].

\section{Classical Texts}

Classical texts provide data which explain this territorial "anomaly" of zuyangming transiting through yin areas.

Su Wen 8 narrates the dialog between Huang Di and Qi Bo describing how is this set arranged; it is an interdependent set form by the six fu (viscera) and the five zang (organs) totaling eleven elements although twelve are mentioned: heart, lung, liver, gall bladder, tanzhong, spleen, stomach, large intestine, small intestine, kidneys, sanjiao, bladder. When we join spleenstomach together they are reduced to eleven.

The bond between the spleenstomach pair is unique because they share dynamism: rise and fall, reception and distribution, dryness and humidity whereas all the other zangfu perform only one function for each viscus. Spleenstomach being in the sixth place of the organic list of eleven, ranks in the central position (the earth) that is to say the center framed by five function-organs from above and other five from below leaving the spleen quite close to the yang function of the six fu. It is interesting to note that spleenstomach is needed for zangfu, yinyang, meridians, blood, essences and qi renewals, that is why earth in central place means that it does not take a special place but it is in all places which can be hinge or pivot between two qualities of qi [13].

The same Chapter 8 of Suwen [14] states that spleenstomach constitutes the silo and the barn from where the five flavors will emerge. Spleenstomach collects and distributes as well, without losing sight that between these two movements the transformations—digestion and assimilation—are developed.

Let us remember that in this enumerative list, the fifth place is for tan zhong, the sea of energy, where xuli, great stomach luo, pours. Tan zhong constitutes a knot of life in the center of the chest, a concentration of qi energy that gets distributed along all the meridians; this task explains why tanzhong (nurtured by xuli, great stomach luo) is in the fifth position, the center place, unity-meeting of life. It makes sense that piwei is in the sixth place because six corresponds with the maintenance of life. The sixth place, in this case the one in the middle, is to nurture the six fu from an already established space. Number six is what shares, it is the organized flow.

The dynamism of spleenstomach is similar to that of cang lin storage places and barns, as can be read in $\mathrm{Su}$ Wen as we mentioned above. It is interesting to decipher the meaning of these two sinograms, understanding them will bring us closer to the spleenstomach unitary function we are talking about here: cang is the place where a large amount of grain is placed, the silo, as in a gathering, a meeting, a being together, the grain is arranged for its shared cooking and in taking [15].

Lin is the place where the grain is taken (barn) to be kept and then distributed, a double process: collect from distribute to. And this double process (rise and fall) also represents the double aspects of the piwei pair. Surely, spleenstomach, a pair that balances in the central area (middle triple warmer) constitutes the best example of the earth-heaven interrelation of organs, of the earth-heaven dialectic within a visceral set.

And from this singularity we know that stomach wei is basically [16] yang, a fu which transforms and transports towards others fu and that spleen bi is a zang that storages and produces essences to be distributed, and it is added to the fu group because of its capacity to transform, promote, distribute and transport like the five fu but in a zang manner: storing and elevating the clear and pure thus called "root of storages and barns". For Chinese medicine, a zang rather than being a visceral mass or an organ is a movement, a dynamic, a quality of energies, one of the ways of running one of the five polarities needed for the establishment and the maintenance of existence, one of the five wu xing phases.

Cang lin expresses the double task of collecting and distributing to the desired place whenever it is needed. The spleen distributes the afflux of body liquids, essences and nurturing elements toward flesh and towards the 4 limbs which host the twelve meridians trajectories, that is to say to the whole body. This double aspect function is unique and helps us to understand that spleen works as a zang storing and elaborating the essences which then 
distributes to the whole body and that stomach has a fu task transforming and transporting to other $\mathbf{f u}$. Thus spleenstomach is a center between the exterior and the interior with the help of other viscera, because there is no dynamism that can work by its own.

According to Nei Jing theory, yang rises and forms the heaven and yin descends to form the earth. This definition can help us to clarify the "biology" of the meridians whose external route, generally more or less vertical, show us a route, let us say only material, because what is important is the direction in which the energy circulates inside them, the content and the sense of circulation in the paths rather than the "pipelining", that is to say the dynamics of it, the function.

We mentioned above that wei is mainly yang in its function and thus its energy descends. The Chinese thought develops the concept of complementary opposite which, in essence, is telling us that a yang element carries in itself ( and seeks to carry) a portion of yin, being the reverse also true. So, yang stomach has a yin component, the spleen, its pair, which it interchanges and shares functions with. The stomach meridian zuyangming starts in the head and descends until the feet, yang route that, in part, runs through yin territory. The spleen meridian zutaiyin starts in the feet and ascends along the inner face of the leg until the thorax, yin route and territory. Along the road they relate and interchange energy through their functions. Should this be possible if zuyangming circulated in the body in an "orthodox" way through the back or flank, both yang territories?

In Suwen 9 it is assured that the spleen together with the stomach and intestines (large and small) plus the triple heater and the bladder constitute the roots of silos and barns, the residence of the reconstruction [17]. At its base, all depends on taiyin, freely communicated with the earthy energies. Spleen is zang, but this description places it in the fu group and so spleenstomach represents a unit which does not appear in other organic pairs. This unit which differentiates spleenstomach is also expressed in its meridians that run very close in thorax and abdomen, despite of the different zang fu categorization of the organs they represent and the different circulatory direction. Spleen ascends and stomach descends.

The stomach is a yang store and barn: it receives food, breaks it into nutritious essences which still in the process of differentiating the five flavors, get sent to the spleen, it also commands the descent of waste to be eliminated through the intestines, sanjiao and bladder. It is fu but it also works as a zang because it absorbs, stores and processes. Stomach fu and spleen zang, yinyang pair of complementary opposites, show-in my opinion-more complementarity than opposition.

Su Wen 29 mentions the biao li obverse-reverse relation of zutaiyin-zuyangming because the spleenstomach is not an outside-inside bond (nei-wai) but the dynamism which facilitates the connections between the inner and the outer. The inner is the heaven in each of us which we do not have access to, and the outer is appearance and everything around it, the world [18]. It is important to understand the difference between nei-wai and biao-li, complex perhaps but very enriching. The physical has a external wai space to its disposal and everything not related to space is inner or interior, nei. They are yin-yang forms, spleen and stomach beat together, and because of that Suwen 8 presents them as spleenstomach. They are very close together despite being different, or maybe just because of that, one is yin and commands the lower part and the other one, yang, commands the upper part, this could seem opposite to the ascent-descent movement of each one which actually refers to the movement of empting and filling, filling and emptying, one after the other as is the case with the annual seasons: in springsummer yang ascends and yin descends and in fall-winter yin ascends and yang descends. This dynamism is specifically represented in the body by the complementary alternation of the spleenstomach function, prototype of the yinyang link, heavenearth. The only way to put heaven and earth in contact is that heaven descends energies and earth ascends them. In this topic at hand, the earth is represented by the stomach that rules the centrifugal dynamism of descent and by the spleen which commands the centripetal movement of ascent.

This was already announced by Lao Zi in Dao De Jing [19] II

...high and low determine one another, but always through wuwei, that is without a manifested "action," because if it were product of a voluntary acting it would mean that something descended because the opposite ascended.

In the trunk, the diaphragm determines the boundary between upper yang and lower yin (yang above and yin below) and also the lumbar spine in some cases, the sacrum can have a yin quality in spite of belonging to a yang space. The interior or inner side of the body corresponds to yin and the exterior one to yang.

In the trunk, zuyangming (St. earth phase) runs through yin territory along a path parallel to the one of zushaoyin (K. water phase) and not far from zujueyin (Liv wood phase). Registered is the anecdote of doctor Liu 
Zi who, when treating a patient, missed the stomach meridian and mistakenly jabbed the needle into the liver one, quite close in thorax and abdomen [20].

In the face, stomach meridian is clearly located in the less yang area where it controls all the sensory organs. Also in the leg it gets close to yin, considering the tibia edge as a border line between the yin and yang areas.

In the energy levels distribution, yangming is the innermost of the external and carries its strength and brightness to the yin. Its connection with GV.14 reinforces its yang which decreases from top down, and is needed by St. in its exchange with yin spleen and it is also needed to supply Large Intestine, xuli (its great luo vessel) and tangzhong, to nourish the lung and also to make energy available for its long route.

Stomach, sea of liquids and cereals, is one of the four seas: brain is sea of marrows, tanzhong is sea of energy and chong mai, sea of the twelve meridians and the blood but...attention, please! in the series of seas stomach is the only fu, all the rest are zang, that is yin.

All these connections demonstrate how difficult is to talk of one meridian alone for it is never isolated: its raison d'être is to belong to an energy network.

Knowing the energy network the meridians weave to establish deep interorganic relationship, allows us to understand the inseparable links between organs, viscera, meridians and their functions, so constituting an indivisible whole. This body framework is a characteristic approach of Chinese Medicine which addresses the body as a unit where each part represents the whole and vice versa, not giving the anatomical either/or functional division that Western medicine describes isolating ones from others and hence, their functions.

The net or mesh meridians (and the whole organism is a meridian) weave allows to maintain the organic functional union, there are no individualities, everything is one, unity prevails.

Yangming is yang, powerful and bright like the sun. Its function is not exteriorized but, on the contrary, it closes towards the interior to penetrate the yin to which it brings its richness and splendor. This movement explains why, in texts, yangming is frequently described as the "interior of the exterior" of the body: the digestive body tract is exterior because it receives food and expels the rest outside after digesting it; yangming (stomachlarge intestine) is the level in which the ingested solids and liquids are taken to the yin organs to be transformed. The intermediate step to do that is regulated by the hinge level shaoyang (GB-TW) which joins the digestive process in toto where yangming represents movement and homeostatic balance in the internal digestion paths.

\section{Complementary Opposition.}

Shan Hang Lun classic text says that yangming interiorizes towards the yin as much energy as blood because it corresponds to the deepest level of the yang meridians and offers its strength and dynamism: moving, processing, moving the chyle forward, absorbing essences. For all of these, it needs taiyin (Sp) which, in association with humidity balances out yangming affinity for dryness. Taiying and yangming deal with the delicate and labile stability that characterizes the digestive process and, generally, the whole organism. Yangming, with plenty of blood and energy contributes to dry the organs up and prevent humidity which is not only the impregnation of fluids but also a temperature parameter: humidity is cold, dryness is hot therefore these two yangming-taiyin energies balance out each other.

Yangming is one of a dry-hot nature and does not want dryness. Taiyin (cold and humid) does not want humidity and is afraid of humidity because it is rooted in earth and in humidity itself, and so it is understandable that desires some dryness, the one its pair, the stomach, can give. Humidity affects circulation, blocks it, this explains why the spleen cannot carry on with its task of transport and distribution. At flesh level, humidity produces edema and flabbiness.

At the same time, stomach escapes from dryness because its own dry nature needs humidity and liquids to perform its fermentation, maceration and transformation tasks. Suwen 4 summarizes saying that spleenstomach are not very cold, not very hot, not very dry and not very humid.

In this dynamism, we must not forget the participation of chongmai meridian which ascends from St.30 joined to the kidney meridian making use of the solid and liquid substances which gastric physiology provides.

\section{Conclusions}

There is a territorial categorization that governs the network of the meridians paths.

Thorax, abdomen, and pelvis (thoracoabdominal region) host almost the totality of yin organs and yang viscera and all the yin foot and hand meridians run the area, and this circumstance gives a yin energetic predomin- 
ance to the area.

I understand that zuyangming in its shinning condition takes its breath to areas with yin predominance so as to maintain dynamic balances. Stomach is the sea of liquids and cereals, yin material. That is to say, that zuyangming with its route of shinning yang re-harmonizes the yin excess in the mentioned territory.

According to the description of the meridian internal routes made by classic medicine doctors, membranes joining stomach and spleen keep the unity of those which thus fulfill their mission, described since ancient times. They are well defined functions executed by spleenstomach that share yinyang activities: receive, process, store, dry, and moisten.

Nowadays, a decisive role among the action ways of acupuncture is attributed to the connective tissue in its wide variety (membranes included). This does nothing else but confirms the capacity of observation and syncretism of ancient Chinese scholars.

These certainties and reviews transmitted by the Chinese classics, portray, explain what is perceived in the interior of the body (anatomy) and what is acknowledged in organic systemic functions (physiology) in accordance with the role of each organ and each organic pair, always according to the Chinese concept of organism.

Anyway, the spleenstomach exception confirms the yinyang territorial categorization rule for the meridians trajectories according to the functions of the zang fu whose energies they drive.

\section{References}

[1] Peluffo, E. (2013) Medicina China. Claves Teóricas. Miraguano Ediciones, Madrid, 105.

[2] Milsky, C. and Andrès, G. (2009) Traduction \& Commentaires Ling Shu. Edition La Tisserande, Paris.

[3] Matsumoto, K. and Birch, S.G. (1988) Hara Diagnosis: Reflections on the Sea. Paradigm Publications Brookline Massachussetts, 53.

[4] Matsumoto, K. and Birch, S.G. (1988) Hara Diagnosis: Reflections on the Sea. Paradigm Publications Brookline Massachussetts, 49.

[5] Unchuld, P. and Tessenow, H. (2011) Huang Di Nei Jing Su Wen. Annotated Translation. University of California Press, Berkeley Los Angeles, 490.

[6] Veith, I. (1966) The Yellow Emperor’s Classic of Internal Medicine. University of California Press. Berkeley, London, 235.

[7] Matsumoto, K. and Birch, S.G. (1988) Hara Diagnosis: Reflections on the Sea. Paradigm Publications, Brookline, 153.

[8] Major, J.S., Queen, S.A., Meyer, A.S. and Roth, H. (2010) The Huainanzi. Columbia University Press, Columbia.

[9] Rickett, W.A. (2001) Guanzi Study and Translation. Cheng \& Tsui Company, Boston, Worcester.

[10] Needham, J. (1969) La science Chinoise et l’occident (Le grand Titrage) Éditions du Seuil.

[11] Cheng, A. (2002) Historia del Pensamiento Chino edicions bellatera Barcelona.

[12] Lu, H.H., Wang, Y.N., Song, Y.Y. and Lu, J. (2013) The Easter Cultural Signature of Traditional Chinese Medicine: Empirical Evidence and Theoretical Perspectives. Chinese Medicine, 4, 79-86. http://scirp.org/journal/cm http://dx.doi.org/10.4236/cm.2013.43012

[13] Larre, C. and de la Vallée, E.R. (2004) Spleen and Stomach. Monkey Press, 117.

[14] de la Vallée, E.R. et Larre, C. (1993) Suwen Les Once Premiers Traités. Maisonneuve Moulins-lès-Metz (France).

[15] Larre, C. and de la Vallée, E.R. (2004) Spleen and Stomach. Monkey Press.

[16] Porkert, M. (1974) The Theoretical Foundations of Chinese Medicine. Systems of Correspondence. The MIT Press, Cambridge, Massachusetts and London England.

[17] de la Vallée, E.R. et Larre, C. (1993) Suwen. Les Once Premiers Traités. Maisonneuve-Moulins-lès-Metz (France).

[18] Larre, C. and Rochat de la Vallée, E. (2004) Spleen and Stomach. Monkey Press.

[19] Waley, A. (1958) The Way and Its Power. A Study of the Tao Tê Ching and Its Place in Chinese Thought. Grove Weidenfeld, New York.

[20] Lu, G.-D. and Needham, J. (2002) Celestial Lancets. A History of Rationale of Acupuncture and Moxa. Routledge Curzon, London. 